\section{Entorno epidemiológico y respuesta a la epidemia del VIH en Bolivia}

\author{
Juan Pablo Protto, ${ }^{1}$ \\ Diddie Schaaf, ${ }^{2}$ Marco Fidel Suárez ${ }^{3}$ \\ y Christian Darras ${ }^{4}$
}

Forma de citar: Protto JP, Schaaf D, Suárez MF, Darras C. Entorno epidemiológico y respuesta a la epidemia del VIH en Bolivia. Rev Panam Salud Publica. 2008;23(4):288-94.

Palabras clave: VIH, SIDA, epidemiología, servicios de salud, Bolivia.

\footnotetext{
1 Organización Panamericana de la Salud/Organización Mundial de la Salud, Bolivia. Área técnica del Grupo Temático, ONUSIDA. La correspondencia se debe dirigir a Juan Pablo Protto, Organización Panamericana de la Salud/Organización Mundial de la Salud, Bolivia. Área técnica del Grupo Temático, ONUSIDA, Calle Víctor Sanjinés 2678 6to piso, Sopocachi, La Paz, Bolivia. Teléfono: 005912241 2465; fax: 005912241 2598. Correo electrónico: jprotto@bol.ops-oms. org/jpprotto@yahoo.fr

2 Organización Panamericana de la Salud/Organización Mundial de la Salud, Bolivia. Área técnica de Salud ITS VIH/SIDA. La Paz, Bolivia.

3 Organización Panamericana de la Salud/Organización Mundial de la Salud, Bolivia. Enfermedades Transmisibles y Factores de Riesgo. La Paz, Bolivia.

4 Organización Panamericana de la Salud/Organización Mundial de la Salud, Bolivia. La Paz, Bolivia.
}

El VIH/SIDA constituye, actualmente, un problema de salud pública debido a la propagación constante y continua de la enfermedad. La detección temprana de la infección es una estrategia prioritaria en salud pública para la orientación y difusión de los mensajes de prevención, y el tratamiento temprano y oportuno de la enfermedad. En Bolivia, cerca de $50 \%$ del total de personas notificadas al momento del diagnóstico $(n=2190)$ se encuentran en fase SIDA, con una detección tardía de la enfermedad (1).

El objetivo de este trabajo es describir la situación epidemiológica del VIH/SIDA en Bolivia y la respuesta brindada al control de la epidemia hasta fines de 2006. Para ello, se ha realizado un análisis bibliográfico de fuentes nacionales como estudios, boletines e informes de consultorías inéditos, elaborados por las diferentes instituciones y agencias que trabajan en el tema VIH/SIDA en el país como son, entre otros, el Ministerio de Salud y Deportes, los organismos de cooperación internacional como OPS/OMS, ONUSIDA, USAID, los organismos no gubernamentales como Sexsalud, las organizaciones de la sociedad civil (de personas que viven con VIH/SIDA como REDBOL y UNINBOL, y de la comunidad gay, lesbianas y travestis como Adesproc-Libertad) y otras fuentes.

La OPS y ONUSIDA han tipificado la epidemia del VIH/SIDA en tres niveles: incipiente o de bajo nivel, concentrada y generalizada (2). Dicha tipificación está basada en la prevalencia del VIH en mujeres gestantes de zonas urbanas y en grupos con comportamientos de mayor riesgo o grupos vulnerables, por ejemplo los trabajadores sexuales comerciales (TSC), los hombres que tienen sexo con hombres (HSH), y los usuarios de drogas inyectables. Se denomina epidemia incipiente cuando la prevalencia en gestantes de zonas urbanas y en grupos vulnerables es inferior a $1 \%$ y $5 \%$, respectivamente. La epidemia concentrada se refiere a una prevalencia inferior a $1 \%$ en gestantes de zonas urbanas y superior a $5 \%$ en grupos vulnerables; finalmente, se habla de epidemia generalizada cuando la prevalencia en gestantes es mayor a $1 \%$ y en los grupos vulnerables es superior a $5 \%$. En esta última tipificación de la epidemia, el VIH se encuentra bien establecido en la población.

\section{Características de la epidemia del VIH/SIDA en Bolivia}

En Bolivia la epidemia del VIH/SIDA es de tipo concentrada, con prevalencias por encima de 
$5 \%$ en poblaciones vulnerables, principalmente en grupos poblacionales de HSH $(1,3,4)$. Los casos acumulados de VIH/SIDA notificados en el país entre enero de 1984 y octubre de 2006, ascienden a 2190 (figura 1): 1239 son casos acumulados de VIH y 951 de SIDA, con un subregistro nacional estimado mayor a 70\% $(3,5)$. Para fines de 2006, ONUSIDA había estimado que 6700 personas en Bolivia vivirían con el VIH/SIDA $(1,6)$. Los departamentos más afectados y con la mayor notificación de casos en el país, sobre todo a expensas de sus ciudades capitales, son Santa Cruz, Cochabamba y La Paz. La vía de transmisión predominante es la sexual, con más de $90 \%$ de las notificaciones. La relación de casos de VIH/SIDA hombre mujer notificada es superior a 2, es decir, hay una mujer por cada dos hombres notificados $(1,3)$. Si bien se puede suponer una feminización de la epidemia, ya que la relación hombre mujer ha disminuido considerablemente en los últimos años (al inicio de la epidemia era 9:1), es importante destacar que la oferta de pruebas rápidas de VIH a las mujeres embarazadas y a las TSC es mayor que la hecha a los hombres.

La prevalencia de VIH en grupos poblacionales de HSH en Bolivia es de $12,8 \%(1,5,7)$, y en el departamento de Santa Cruz se observa la mayor prevalencia en HSH (cuadro 1). La prevalencia en TSC informada a través de los Centros Departamentales de Vigilancia y Referencia de ITS/SIDA (CDVIR) y de otros estudios de vigilancia epidemiológica realizados en el país, es inferior a $0,5 \%$ ( 1 , $3,5,8)$. Se dispone de escasa información sobre el impacto del VIH/SIDA en ciertos grupos poblacionales, pero se calcula que el VIH está llegando a grupos que no han sido tomados en cuenta por la política nacional, como son las personas de la calle, los clientes de los TSC y la población rural (prevalencia estimada: $0,2 \%, 3,5 \%$, y $0.05 \%$, respectiva- mente) (5). En Bolivia la epidemia ha alcanzado a todos los estratos socioeconómicos, abarca a todas las edades y ha penetrado el ámbito rural.

Desde 2000 funcionan siete sitios para el estudio centinela del VIH/SIDA dirigido a poblaciones de bajo riesgo para $\mathrm{VIH}$; se trata de centros hospitalarios y maternidades de El Alto, La Paz, Cochabamba, Santa Cruz, Tarija, Guayaramerin y Cobija. En cuatro de estos sitios se realiza la vigilancia centinela a mujeres embarazadas y en tres se vigilan otros grupos de la población general. Las prevalencias notificadas en estos grupos poblacionales han sido consistentemente inferiores a $1 \%(1,3,5$, 9). A partir de la información disponible, se estima que es muy baja la cobertura de los servicios públicos de diagnóstico y atención para poblaciones HSH $(<5 \%)$ y TSC $(<30 \%)(3,5)$. Por este motivo, estos grupos son considerados de alta importancia y vulnerabilidad.

\section{Respuesta nacional a la epidemia}

El modelo actual de atención pública para personas que viven con el VIH/SIDA (PVVS) muestra signos de fragilidad. Actualmente existen 13 CDVIR en Bolivia; son centros públicos auspiciados por la Agencia de los Estados Unidos para el Desarrollo Internacional (USAID) hasta finales de 2006, orientados a brindar atención a PVVS. Sin embargo, desde un inicio también atendieron a TSC, de tal manera que se estima que $85 \%$ de los servicios de los CDVIR han sido para TSC registrados, con una limitada atención específica a HSH y a PVVS. La estigmatización de estos centros y la falta de respuesta nacional hacia una descentralización de la atención del VIH a centros hospitalarios especializados e interdisciplinarios, determina que la

FIGURA 1. Notificación de casos de VIH/SIDA. Bolivia, enero 1984-octubre 2006

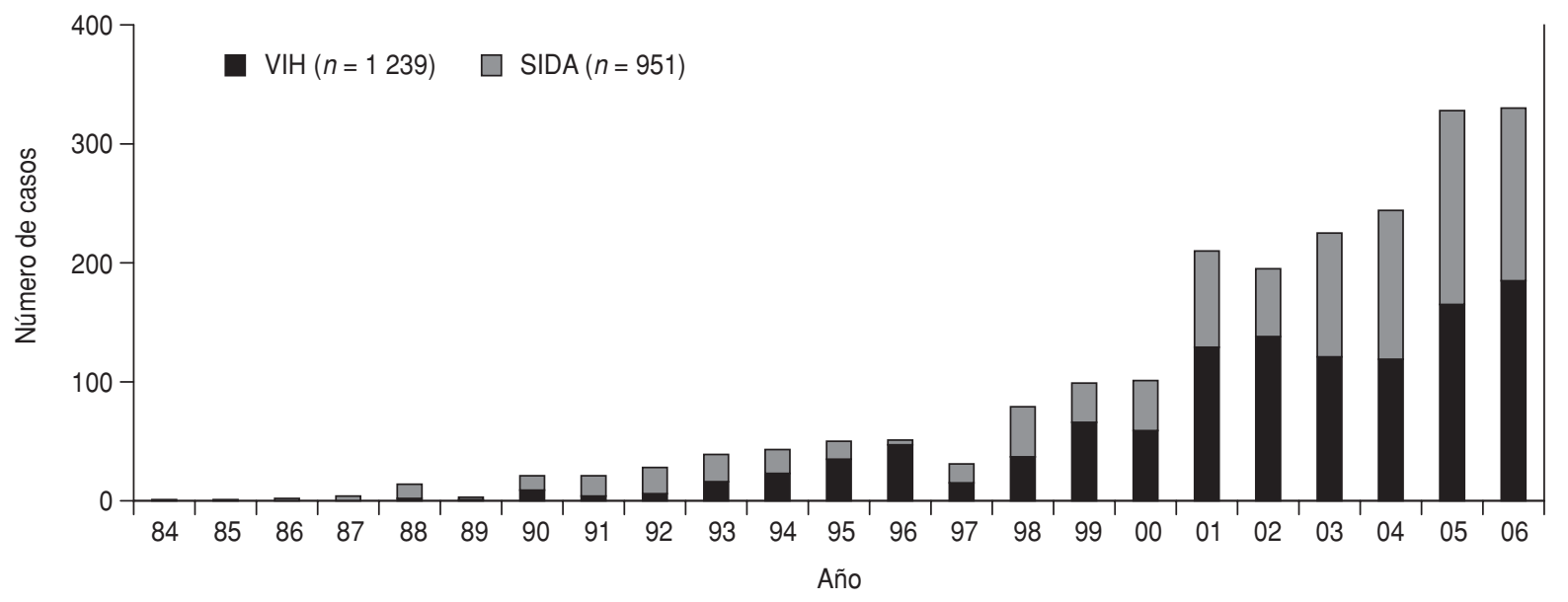


CUADRO 1. Estudios de prevalencia del VIH en hombres que tienen sexo con hombres (HSH) de Bolivia

\begin{tabular}{lcccr}
\hline \multicolumn{1}{c}{ Fuente, ciudad y año } & $\begin{array}{c}\text { Personas } \\
\text { tamizadas }\end{array}$ & $\begin{array}{c}\text { Número de } \\
\text { positivas }\end{array}$ & Prevalencia \% & IC95\% $^{\text {a }}$ \\
\hline Bartoloni. Santa Cruz, 1987 & 12 & 0,00 & 0,00 & $0,00-30,13$ \\
Melgar. Santa Cruz, 1988 & 68 & 7 & 10,29 & $4,59-20,65$ \\
Hierholzer. La Paz, 1999-2001 & 48 & 7 & 14,58 & $6,54-28,38$ \\
NMRCD. Santa Cruz, 2001-2002 & 238 & 52 & 21,85 & $16,88-27,75$ \\
\hline
\end{tabular}

Fuente: Bolivia. Ministerio de Salud y Deportes. Categorización de la epidemia de VIH-SIDA en Bolivia. La Paz: OPS/OMS; 2003. a Intervalo de confianza de $95 \%$ para la prevalencia.

atención pública a PVVS no sea integral, periódica y oportuna $(3,5,10,11)$. En Bolivia son escasos los centros privados que brindan orientación y atención a HSH, TSC y PVVS. En la actualidad ninguna estrategia nacional contempla a niñas y niños huérfanos $\mathrm{y} / \mathrm{o}$ en situación vulnerable a causa del VIH/SIDA.

Para el desarrollo e implementación de políticas y estrategias nacionales sostenibles en el área del VIH/SIDA, resulta importante contar con recursos humanos capacitados y comprometidos en el plano gubernamental. Hasta diciembre de 2006, el Programa Nacional de ITS/VIH/SIDA, como parte de la estructura técnica y administrativa del Ministerio de Salud y Deportes, contaba con 117 profesionales técnicos distribuidos en los nueve departamentos del país $(1,9)$, con recursos provenientes del gobierno y de la cooperación internacional. Los médicos especialistas capacitados y con experiencia en el manejo clínico de las PVVS son escasos. Hasta la fecha no se ha elaborado ningún protocolo de atención sobre el manejo clínico del $\mathrm{VIH} / \mathrm{SIDA}$ en el plano nacional. Estudios realizados por las organizaciones de PVVS del país indican que $83.8 \%$ declaran tener necesidades que no son atendidas por los servicios de salud, además de la ausencia de médicos especialistas con enfoque interdisciplinario, la baja atención de calidad y calidez brindada en los servicios de salud, el escaso apoyo nutricional prestado desde los centros de atención a PVVS y los problemas en la entrega de antirretrovirales (ARV) y medicamentos para enfermedades oportunistas $(12,13)$.

En Bolivia se estima que sólo $4 \%$ de las mujeres embarazadas y con VIH reciben el ciclo completo de profilaxis para evitar la transmisión vertical. Hasta octubre de 2006 se había notificado que 337 PVVS recibían terapia ARV. Empero, estimaciones de OPS/ONUSIDA consideran que son 1600 PVVS las que necesitarían contar con tratamiento $(3,5)$. Por otra parte, la adherencia al tratamiento es un obstáculo para las PVVS: una de cada tres menciona no recibir la terapia ARV por diversas razones como son el miedo a la toma de los medica- mentos, el consumo de alcohol, los efectos adversos provocados por los propios ARV (más evidenciado en mujeres), la falta de pruebas de laboratorio para hacer el seguimiento y evolución de la enfermedad $\mathrm{y}$, finalmente, la poca disponibilidad de ARV (12, 13). La no adherencia al tratamiento predispone al desarrollo temprano de resistencia viral y a la limitación en las opciones disponibles para futuras combinaciones de drogas antirretrovirales.

En cuanto a las enfermedades oportunistas más prevalentes, el Programa Nacional de ITS/ VIH/SIDA carece de datos nacionales o departamentales, pero se estima que $17 \%$ de las PVVS cursan anualmente con alguna. En el plano regional, la tuberculosis, las neumonías por $\mathrm{N}$. carinii, el citomegalovirus, el sarcoma de Kaposi y las candidiasis, son los padecimientos más prevalentes (5, 14-16). A través de estudios realizados por organizaciones de PVVS, se observa que en Bolivia las diarreas graves, la tuberculosis y las candidiasis constituyen las patologías más frecuentemente declaradas por las PVVS $(12,13)$.

El acceso y la cobertura del tratamiento con ARV y de las enfermedades oportunistas continúa siendo un problema en Bolivia, atribuido a diversos factores como son: la excesiva burocracia administrativa en los distintos niveles gubernamentales; la falta de implementación del Sistema Administrativo Logístico de Medicamentos e Insumos en los CDVIR; las demoras en la distribución de los medicamentos y reactivos; la falta de control y seguimiento de las regionales y del nivel central de los insumos enviados y recibidos y, por último, las debilidades gerenciales nacionales y regionales (Programas departamentales y responsables regionales de los centros de almacenamiento-CEASS)(1).

En el país existen aproximadamente 25 bancos de sangre y/o servicios de transfusión que realizan pruebas de VIH, y tres laboratorios de referencia que se encargan de la confirmación de las pruebas positivas y de las pruebas de seguimiento de la infección VIH y el SIDA, como conteo de CD4/CD8 y cargas virales (9). El tamizaje de la sangre para detectar agentes causales de enfermedades que se 
transmiten por vía sexual o bien sanguínea (sífilis, hepatitis, VIH, etc.) ha tenido un impacto positivo en el control de la transmisión de estos padecimientos. En la actualidad menos de $1 \%$ de los casos acumulados han contraído la infección por la vía sanguínea. En 2004, la prevalencia observada en el tamizaje de VIH de 40430 donantes fue de $0,13 \%$ (5).

En lo que concierne al marco legal, hasta la fecha no existe una ley nacional de SIDA que regule la prevención y atención integral del VIH y proteja los derechos de las PVVS. Desde hace más de cinco años, en el Congreso Nacional se encuentra un proyecto de ley para ser aprobado y promulgado. Únicamente existen resoluciones (como la 0711) que contemplan el respeto de ciertas pautas legales en materia de atención VIH/SIDA, sobre todo en lo referente a promover la prueba de VIH con consentimiento informado, la confidencialidad y la defensa ante la discriminación a personas con VIH/SIDA. Lamentablemente Bolivia no cuenta con puntos focales en las instituciones gubernamentales para la vigilancia de los derechos humanos relacionadas con el VIH/SIDA; asimismo, carece de mecanismos de seguimiento y recolección de información sobre derechos humanos, además de que no se han desarrollado procesos de sensibilización sobre VIH/ SIDA y de derechos humanos dirigidos a autoridades del poder judicial $\mathrm{y}$, por último, tampoco existen servicios de apoyo jurídico para casos individuales relacionados con el VIH/SIDA (1). Los mecanismos o instancias legales comúnmente utilizados frente a actos o acciones que violan los derechos humanos de las PVVS, son las denuncias presentadas a la defensoría del pueblo y a abogados privados. Las herramientas legales que existen hoy en día en el país son los amparos constitucionales como el Artículo 7 de la Constitución Política del Estado y las resoluciones ministeriales y/o defensoriales $(17,18)$. En 2005 y 2006 se presentaron seis querellas a la defensoría del pueblo por parte de PVVS. En 2002, las redes de PVVS de Bolivia presentaron un recurso de amparo a la Comisión Interamericana de Derechos Humanos, a fin de garantizar la provisión de ARV en el país; en 2003 la Comisión falló a favor de la demanda, lo cual llevó al Estado a generar garantías en cuanto a la compra y el abastecimiento de antirretrovirales.

El aporte financiero brindado por la cooperación internacional a políticas y programas de $\mathrm{VIH} / \mathrm{SIDA}$ representa $84 \%$ del presupuesto global nacional destinado a este padecimiento, siendo la contribución del gobierno de tan sólo 16\% (1). Desde 2002, mediante convenios de cooperación entre los gobiernos de Brasil-Bolivia, el país recibe apoyo para la implementación de centros especializados en el manejo de las infecciones de transmisión sexual (ITS) y el VIH/SIDA, la capacitación del
CUADRO 2. Costos estimados de acceso universal a la atención de VIH/SIDA, Bolivia, 2006

\begin{tabular}{lr}
\hline Líneas de intervención & $\begin{array}{r}\text { Total costos } \\
\text { (en dólares) } \\
\text { Escenario 100\% }\end{array}$ \\
\hline Prevención en grupos vulnerables & 5530893 \\
Hombres que tienen sexo con hombres & 4265808 \\
Trabajadoras sexuales & 1134347 \\
Clientes de trabajadores sexuales & 63418 \\
Personas que viven en la calle & 67319 \\
Personas con VIH/SIDA & 8536469 \\
Prevención positiva & 659142 \\
Quimioprofilaxis & 12256 \\
Infecciones oportunistas & 91944 \\
Tratamiento antiretroviral de gran actividad & 7773127 \\
Transmisión vertical & 2400082 \\
Madre & 1544366 \\
Niño & 855716 \\
Prevención en población general & 9459593 \\
De 10 a 17 años & 2055355 \\
De 10 a 49 años & 7404238 \\
Servicios sistema de salud & 7060314 \\
Sangre y hemoderivados & 5321750 \\
Recursos humanos & 1506194 \\
Infraestructura & 232370 \\
Monitoreo & 2449368 \\
Gran total & 35436718 \\
\hline
\end{tabular}

Fuente: Programa Conjunto de las Naciones Unidas sobre el VIH/SIDA. Plan para alcanzar el acceso universal a la atención integral en Bolivia 2006-2010. La Paz: ONUSIDA; s.f.

personal en el manejo clínico de las personas con VIH/SIDA y medicamentos ARV de primera línea. En 2005 el Fondo Global contribuyó con la compra de reactivos para el diagnóstico y seguimiento de las ITS y VIH/SIDA, medicamentos para el tratamiento de las ITS, antirretrovirales de segunda línea para el VIH/SIDA, y tratamiento para enfermedades oportunistas $(1,5)$.

Según ONUSIDA, el costo estimado en Bolivia para lograr una cobertura total en materia de acceso universal a la prevención, atención y tratamiento del VIH, es decir la disponibilidad continua de servicios de atención integral por parte del público, independientemente de su situación legal o de su capacidad económica, asciende a 35,4 millones de dólares; el costo estimado para implementar la prevención en grupos vulnerables es de 15,9\% (5) (cuadro 2).

Los mecanismos de coordinación multisectorial, encabezados por el gobierno para hacer seguimiento y monitoreo de las políticas y estrategias VIH implementadas en todo el país, son escasos (1). El Grupo Temático Ampliado de Naciones Unidas para el VIH y el Mecanismo de Coordinación País para el Fondo Global, constituyen las dos instancias nacionales de coordinación en la temática VIH. 


\section{Factores determinantes asociados a la propagación del VIH}

Bolivia es un país multiétnico y pluricultural que sufre de migraciones múltiples y temporales desde el área rural hacia lugares donde se pueden encontrar fuentes de trabajo. Los procesos migratorios, sumados a factores tales como el machismo, el consumo excesivo de alcohol, la falta de concienciación y conocimiento sobre el VIH/SIDA y sus formas de transmisión y prevención, los altos índices de violencia y los prejuicios y falsas creencias acerca del tema, favorecen la rápida transmisión de las ITS y el VIH en el país. Es poca la información que existe acerca de los procesos y dinámicas de la sexualidad y sus determinantes en ciertas poblaciones rurales vulnerables, migrantes, que permitan disenar estrategias adecuadas de prevención y atención para disminuir la tasa de ITS y VIH/SIDA, promoviendo cambios de comportamiento. Estudios realizados en comunidades y municipios migrantes de habla quechua, del departamento de Chuquisaca, demuestran que una alta proporción de la población desconoce las ITS/VIH/SIDA y su prevención; se ha observado que el condón es muy poco utilizado y que las prácticas sexuales de riesgo son frecuentes en la población (10).

El considerable estigma y la discriminación reinantes en el país inciden en la propagación de la enfermedad, sobre todo entre las poblaciones vulnerables. A esto se suma la escasez de servicios, por un lado, y la renuencia a acudir a los mismos, por el otro (19). En muchas comunidades no se ofrecen servicios para ITS/VIH/SIDA a los hombres, o bien son insuficientes; en Bolivia los HSH representan aproximadamente $5 \%$ de la población masculina de 15 a 49 años, pero equivalen a $60 \%$ del total de casos de VIH estimados $(1,5)$. Estos factores ocasionan que esos grupos de población no accedan de manera oportuna a los servicios de orientación sobre ITS/VIH-SIDA, a las pruebas y a los servicios asistenciales apropiados (figura 2).

La relación entre el VIH/SIDA y el estigma lleva a muchas personas que están dentro de una norma social, a no considerarse afectados por la enfermedad y a seguir practicando comportamientos inseguros que las ponen en riesgo. Al disminuir la motivación para hacerse las pruebas y cuidar de la salud propia, el estigma y la discriminación promueven la propagación del VIH, con consecuencias personales y sociales graves, además de que pueden tener consecuencias psicológicas profundas en las PVVS, al intensificar el aislamiento social y la depresión (20).

Los datos nacionales ponen de manifiesto la necesidad de elaborar una política nacional intersectorial sostenible en relación con el VIH/SIDA, encabezada y supervisada por el Ministerio de

FIGURA 2. Repercusiones del estigma del VIH/SIDA en el proceso continuo de la prevención. Bolivia, 2003

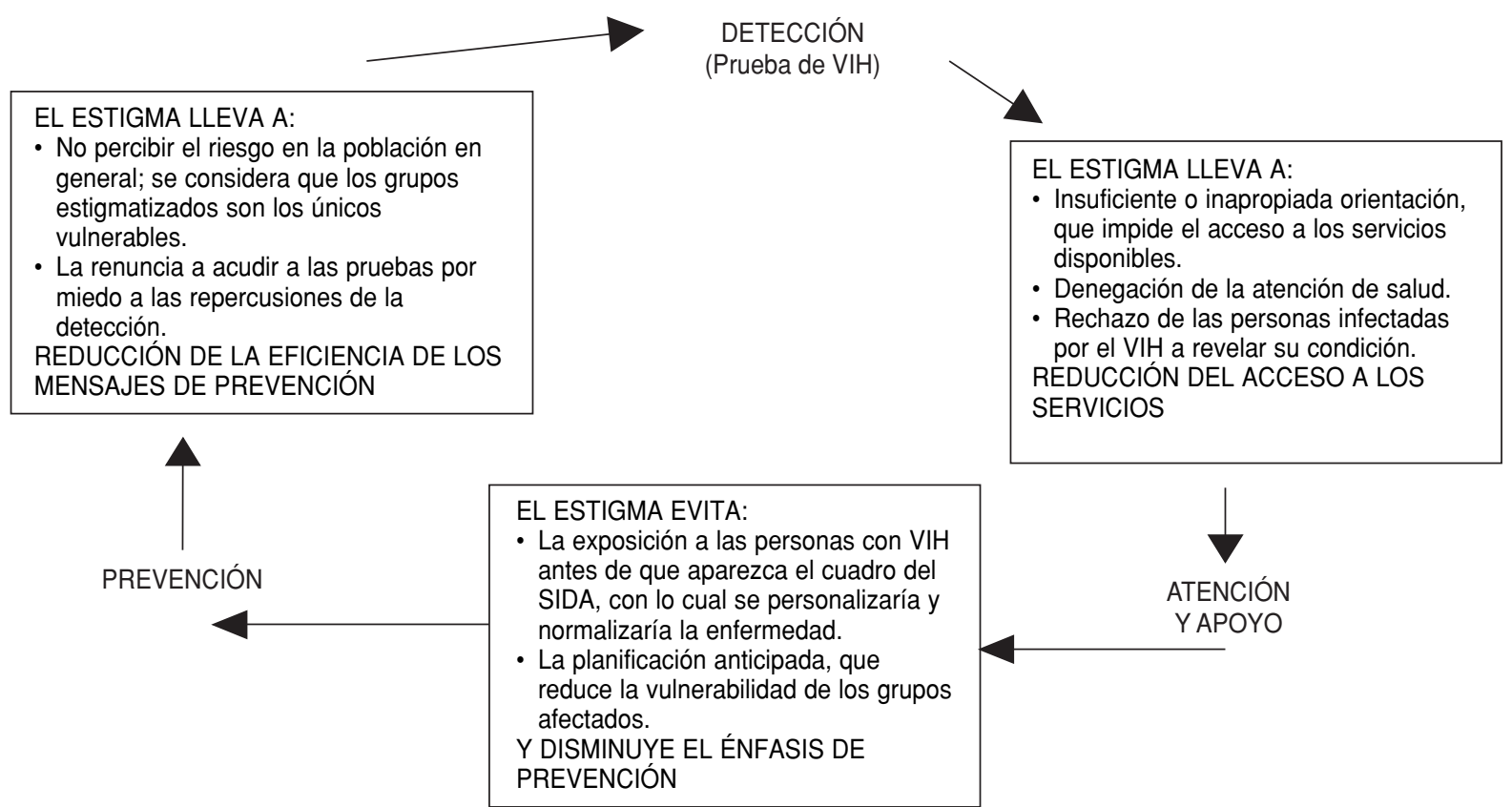

Fuente: Organización Panamericana de la Salud. Organización Mundial de la Salud. Comprensión y respuesta al estigma y discriminación por el VIH en el sector salud. Washington: OPS/OMS; 2003. 
Salud y Deportes a través del Programa Nacional de ITS/VIH/SIDA. Esta política debe contemplar un enfoque intersectorial y estar dirigida a promover, fortalecer y ampliar los programas de prevención y atención del VIH/SIDA del país, garantizando el acceso universal a la atención integral de ITS/VIH/SIDA, con énfasis particular en los grupos poblacionales más vulnerables al riesgo de infección por el VIH.

De acuerdo con las recomendaciones establecidas por OPS/ONUSIDA (21-25), las políticas nacionales relativas al VIH/SIDA tendrían que construirse tomando en cuenta cuatro pilares estratégicos:

1. El diseño e implementación de un solo marco de acción nacional que provea la base de coordinación nacional y departamental, a través de la elaboración de un plan estratégico multisectorial nacional VIH/SIDA, adecuado y pertinente a la realidad del país, que permita determinar los lineamientos, políticas y estrategias nacionales VIH/SIDA de manera armonizada.

2. La conformación de un ente nacional multisectorial VIH/SIDA, liderado por el Ministerio de Salud y Deportes, que haga seguimiento y evaluación nacionales de las metas integrales de control de la epidemia. La conformación de una unidad nacional de monitoreo y evaluación que promoverá el fortalecimiento de la vigilancia epidemiológica nacional del VIH/SIDA a través de la recopilación y el análisis oportuno de la información, normando los indicadores de verificación y control de la epidemia y estableciendo una única estrategia de divulgación y uso de la información.

3. Una sola autoridad de coordinación nacional, con un mandato de amplia base multisectorial que permita armonizar y determinar las políticas de acción y las estrategias de impacto.

4. Un marco legal nacional que vele por el respeto de los derechos humanos de las personas que viven con VIH/SIDA (Promulgación de la Ley Nacional de Sida).
Para lograr la sustentabilidad de las políticas de VIH/SIDA en el tiempo, es necesario contar con un mayor compromiso y participación del gobierno en el abordaje del VIH/SIDA. El mismo debe promover y garantizar estabilidad y capacitación del capital humano, e incrementar la asignación de recursos presupuestarios y humanos hacia el VIH/ SIDA, fortaleciendo la gerencia nacional y regional y la coordinación interinstitucional e intersectorial. Conocer la propia epidemia y comprender los factores que la impulsan, es absolutamente fundamental para la prevención del VIH y lograr una respuesta de largo plazo al SIDA.

\section{SYNOPSIS}

\section{Epidemiological environment and response to the HIV epidemic in Bolivia}

With AIDS/HIV, early detection is of key importance to public health, as well as disseminating prevention information and providing timely and appropriate treatment. In Bolivia, at the end of 2006 approximately 50\% had AIDS at the time of diagnosis, detection having occurred late in the illness. The HIV/AIDS epidemic in Bolivia is concentrated, with prevalence rates over $5 \%$ among the at-risk population, primarily men who have sex with men. From January 1984 through October 2006, the total number of HIV/AIDS cases reported in Bolivia rose to 2 190, with 1239 HIV and 951 AIDS cases, and underreporting estimated to be over $70 \%$ country-wide. The United National Joint Program on AIDS (UNAIDS) estimated that by the end of 2006 there would be 6700 people living with HIV/AIDS in Bolivia. In the context of this scenario, the article describes the challenges facing the HIV/AIDS program and the strategies developed to address the epidemic in Bolivia. In addition, the UNAIDS/PAHO strategies are stressed and must get underway for HIV/AIDS prevention and control activities in the country.

Key words: HIV, AIDS, epidemiology, health services, Bolivia.

\section{REFERENCIAS}

1. Bolivia. Ministerio de Salud y Deporte. Organización Panamericana de la Salud. Diagnóstico situacional, monitoreo y evaluación de la implementación del compromiso UNGASS sobre el VIH/ SIDA en Bolivia. La Paz: ONUSIDA; 2006.

2. Programa Conjunto de las Naciones Unidas sobre el VIH/SIDA. Organización Panamericana de la Salud. Organización Mundial de la Salud. Pautas para la vigilancia de infecciones de transmisión sexual. Washington: OPS/OMS/ ONUSIDA; 1999.

3. Bolivia. Ministerio de Salud y Deportes. Caracterización de la epidemia del VIH/SIDA en Bolivia. La Paz: Boletín Informativo Epidemiológico; 2006.

4. Bolivia. Ministerio de Salud y Deportes. Categorización de la epidemia de VIHSIDA en Bolivia. La Paz: OPS/OMS; 2003.
5. Programa Conjunto de las Naciones Unidas sobre el VIH/SIDA. Plan para alcanzar el acceso universal a la atención integral en Bolivia 2006-2010. La Paz: ONUSIDA; s.f.

6. Programa Conjunto de las Naciones Unidas sobre el VIH/SIDA. Informe sobre la epidemia mundial de SIDA 2006. Ginebra: ONUSIDA; 2006.

7. Bautista CT, Sanchez JL, Montano SM, Laguna-Torres VA, Lama JR, Sanchez 
JL, et al. Seroprevalence of and risk factors for HIV-1 infection among South American men who have sex with men. Sex Transm Infect 2004:80:498-504.

8. Bautista CT, Sanchez JL, Montano SM , Laguna-Torres A, Suarez L, Sanchez J, et al. Seroprevalence of and risk factors for HIV-1 infection among female commercial sex workers in South America. Sex Transm Infect 2006;82:311-6.

9. Bolivia. Ministerio de Salud y Deportes. Programa Nacional de ITS/VIH/SIDA. Plan estratégico nacional de ITS/VIH/ SIDA 2004-2008: versión preliminar. La Paz: s.e.; 2003.

10. Universidad Andina Simón Bolívar. Programa Conjunto de las Naciones Unidas sobre el VIH/SIDA. Estudio de los conocimientos, actitudes y practicas sexuales para las infecciones de transmisión sexual, VIH/SIDA: municipios de Poroma y Tarabuco, Departamento de Chuquisaca. Sucre: UASB-ONUSIDA; 2006.

11. Centers for Disease Control and Prevention. Fortalecimiento del Sistema de Vigilancia Epidemiológica de ITS/VIH/ SIDA del Programa Nacional de ITS/ VIH/SIDA y de los CDVIR. La Paz: CDC/USAID/BOLIVIA; 2004.

12. Red Nacional de Personas que Viven con el VIH y el SIDA de Bolivia. El acceso a la atención integral para las personas viviendo con VIH/SIDA en Bolivia. La Paz: OPS/ONUSIDA; s.f.
13. Bolivia. Unidad Nacional Boliviana Identificación de Necesidades de la Red UNINBOL frente al acceso universal y atención integral de las personas viviendo con el VIH y/o SIDA en Bolivia. La Paz: OPS/ONUSIDA; s.f.

14. Programa Conjunto de las Naciones Unidas sobre el VIH/SIDA. Enfermedades oportunistas relacionadas con el VIH: actualización técnica del ONUSIDA. Ginebra: ONUSIDA; 1999

15. Programa Conjunto de las Naciones Unidas sobre el VIH/SIDA. Plan para alcanzar el acceso universal a la atención integral en Perú 2006-2010. Lima: ONUSIDA; 2006.

16. Gona $\mathrm{P}$, Van Dyke R, Williams $\mathrm{P}$, Dankner W, Chernoff M, Nachman S, et al Incidence of opportunistic and other infections in HIV infected children in the HAART era. J Am Med Assoc 2006;296(3):292-300.

17. Bolivia. Ministerio de Salud y Deportes. Programa Nacional de ITS/VIH/SIDA. Resolución Ministerial N. 0711 para la prevención y vigilancia del VIH/SIDA en Bolivia. La Paz: Ministerio de Salud y Deportes; 2005.

18. Defensor del Pueblo. República de Bolivia. Resolución Ministerial N RD/LPZ/ 00007/2003/DH. La Paz: Defensoría del pueblo; 2002

19. Organización Panamericana de la Salud. Organización Mundial de la Salud. Comprensión y respuesta al estigma y discriminación por el VIH en el sector salud. Washington: OPS/OMS; 2003.

20. Comunidad Andina de Naciones. Informe comparado sobre la situación del VIH/SIDA y los Derechos Humanos en la Comunidad Andina de Naciones. Seguimiento al cumplimiento de las obligaciones emanadas de la declaración de compromiso en la lucha contra el VIH/ SIDA. Lima: CAN; 2003.

21. Programa Conjunto de las Naciones Unidas sobre el VIH/SIDA. Intensificación de la prevención del VIH. Documento de posición de política del ONUSIDA. Ginebra: ONUSIDA; 2006.

22. Programa Conjunto de las Naciones Unidas sobre el VIH/SIDA. Los "tres Unos" en acción: dónde estamos y adónde nos dirigimos. Ginebra: ONUSIDA; 2005.

23. Organización Panamericana de la Salud. Organización Mundial de la Salud. Plan regional de VIH/ITS para el sector salud 2006-2015. Washington: OPS/OMS; 2005.

24. Pan American Health Organization. Toward universal access to HIV prevention, care, and treatment: 3 by 5 report for the Americas. Washington: PAHO; 2006.

25. Organización Mundial de la Salud. ONUSIDA. Progresos realizados en materia de acceso mundial al tratamiento antiretroviral contra el VIH.; Informe sobre "tres millones para 2005" y más adelante. Ginebra: PAHO-ONUSIDA; 2006.

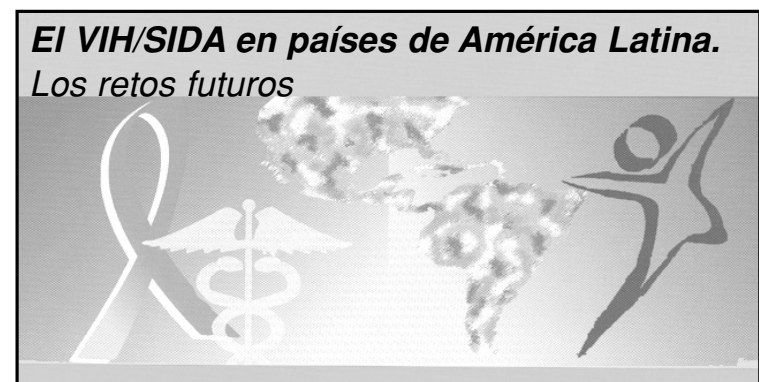

Este libro presenta información reciente y actualizada acerca de la magnitud y tendencias de la epidemia de VIH/SIDA en América Latina. En esta obra se evalúa la capacidad actual de vigilancia en los países, se examinan las respuestas nacionales del sector de la salud a la epidemia en cada país, se identifican las áreas fundamentales en las que se requieren con urgencia intervenciones específicas y se describen los retos futuros.

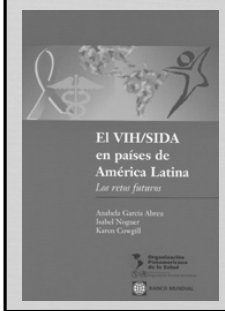

$2004 \cdot x i 316$ pp. • ISBN 9275315973 Código: PC $597 \cdot$ Precios: US $\$ 26.00$ en América Latina y el Caribe/ US $\$ 20.00$ fuera de América Latina

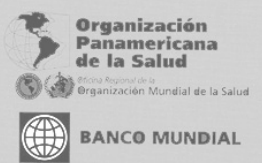

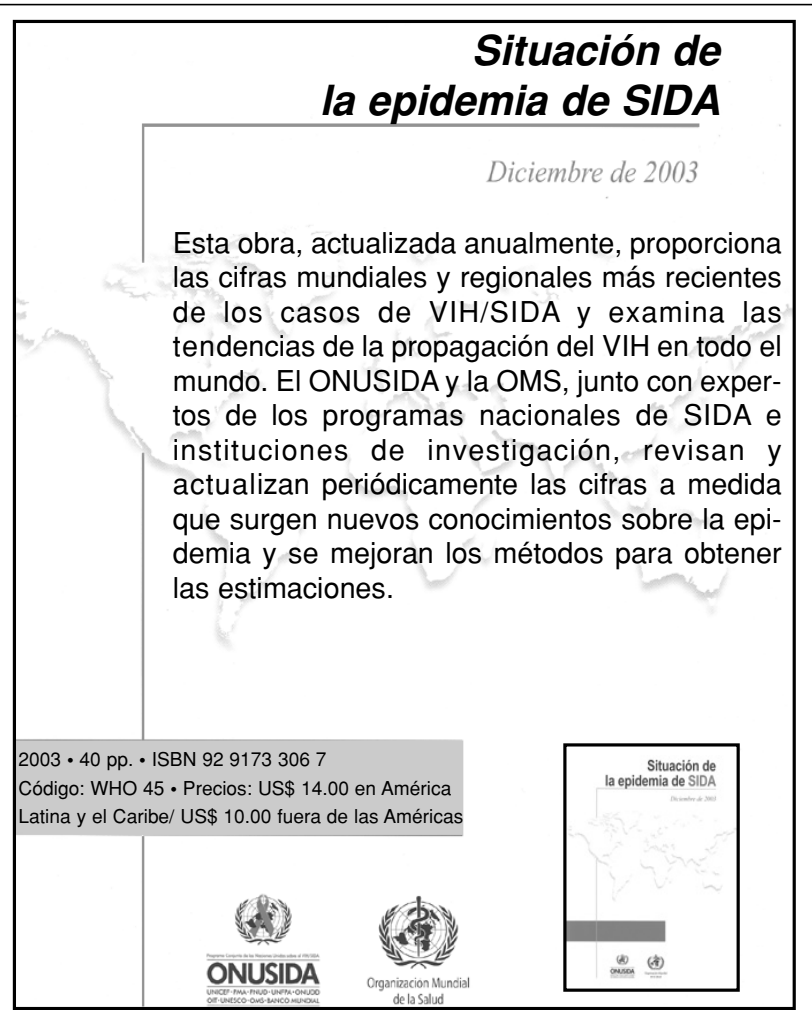

Esta obra, actualizada anualmente, proporciona las cifras mundiales y regionales más recientes de los casos de VIH/SIDA y examina las tendencias de la propagación del VIH en todo el mundo. EI ONUSIDA y la OMS, junto con expertos de los programas nacionales de SIDA e instituciones de investigación, revisan y actualizan periódicamente las cifras a medida que surgen nuevos conocimientos sobre la epidemia y se mejoran los métodos para obtener las estimaciones.

ONUSIDA

Para solicitar estas publicaciones: http://publications.paho.org Fax: (301) 206-9789 • Correo electrónico:paho@pmds.com 